Check for updates

Cite this: RSC Adv., 2018, 8, 17739

\title{
Facile fabrication of highly conductive tracks using long silver nanowires and graphene composite $\uparrow$
}

\author{
Su Ding, (D) *a Luxi Zhang, ${ }^{\text {a }}$ Weitao Su${ }^{* a}$ and Xiwei Huang ${ }^{\mathrm{b}}$ \\ Flexible long silver nanowires and graphene (AgNW-G) hybrid tracks with ultra-low resistivity were prepared \\ by a low-temperature sintering process. The long AgNWs connect the graphene flakes in a plane and \\ establish bridges between graphene layers by building a 3D conductive network, and finally induce \\ a resistivity of $8.6 \mathrm{~m} \Omega \mathrm{cm}$ with a concentration of AgNWs of $20 \mathrm{wt} \%$ and sintering at $150{ }^{\circ} \mathrm{C}$. The AgNW- \\ $\mathrm{G}$ tracks show superior reliability even after 100 bending cycles. The use of AgNW-G tracks in flexible \\ circuits indicates their possible applications in flexible electronics.
}

Received 29th March 2018

Accepted 2nd May 2018

DOI: $10.1039 / c 8 r a 02726 a$

rsc.li/rsc-advances et al. ${ }^{22}$ printed graphene inks on surface pre-treated T-shirts to fabricate graphene e-textiles, which were further applied to collect heart beat signals, showing good performance during cycled bending. Regarding the aforementioned progress, the electrical conductivity of graphene tracks is still too low for application in printed electronics. A promising route to enhance the electrical conductivity is hybridizing the graphene ink with metallic nanoparticles. ${ }^{30-34}$ Tien et al..$^{30}$ synthesized graphene nanosheets decorated with Ag nanoparticles and constructed hybrid conductive films. Ag nanoparticles act as useful spacers and conductors, which effectively reduces the sheet resistance of Ag-graphene films from $8.2 \times 10^{3} \Omega \mathrm{sq}^{-1}$ to $93 \Omega \mathrm{sq}^{-1}$. Ag nanoparticle decorated graphene ink is also employed to fabricate conductive tracks with a low resistivity of $20 \Omega \mathrm{sq}^{-1}$ (with annealing at $400{ }^{\circ} \mathrm{C}$ for 30 minutes). ${ }^{35}$ This indicates that $\mathrm{Ag}$ nanoparticle decorated graphene ink obviously improves the electrical property in contrast to pure graphene inks.

It is supposed that the addition of AgNWs to graphene sheets for the fabrication of conductive tracks will exhibit better conductivity than Ag nanoparticles since the long AgNWs build more effective conductive pathways between the graphene nanoflakes. However, this routine has seldom been reported. Chen et al. ${ }^{36}$ proposed a novel architecture for graphene paper by mixing $1 \mathrm{D}$ AgNWs and graphene sheets produced by chemical vapor deposition (CVD). The electrical conductivity of the obtained free-standing AgNW-graphene (AgNW-G) paper is enhanced to $3189 \mathrm{~S} \mathrm{~cm}^{-1}$, which is superior to that of $\mathrm{Ag}$ nanoparticle/graphene tracks in previous papers. ${ }^{35,37}$ However, the production of CVD grown graphene is too complex and expensive. Chen's group ${ }^{38}$ presented a strain sensor based on AgNWs and graphene hybrid particles via a coprecipitation, reduction, vacuum filtration and casting process. The highest conductivity of the hybrid composite is $107.1 \mathrm{~S} \mathrm{~cm}^{-1}$. The multistep fabrication process, including reduction of graphene oxide sheets, is undesirable. Furthermore, to the best of our
${ }^{a}$ College of Materials and Environmental Engineering, Hangzhou Dianzi University, 310018 Hangzhou, P. R. China. E-mail: dingsu@hdu.edu.cn; suweitao@hdu.edu.cn ${ }^{b}$ Key Laboratory of RF Circuits and Systems (Hangzhou Dianzi University), Ministry of Education, 310018 Hangzhou, China

$\dagger$ Electronic supplementary information (ESI) available. See DOI: $10.1039 / \mathrm{c} 8 \mathrm{ra} 02726 \mathrm{a}$ 
knowledge, systematic studies of the effect of AgNW size and sintering temperature on the performance of the hybrid AgNW$\mathrm{G}$ tracks are still lacking. Herein, we designed a hybrid ink composed of commercial graphene ink and long AgNWs. The hybrid AgNW-G tracks sintered at a low temperature of $150{ }^{\circ} \mathrm{C}$ for only 15 minutes show an ultra-high electrical conductivity. In addition, the AgNW-G hybrid ink was printed on polyethylene terephthalate (PET) substrates to make flexible tracks, which were bent for 100 cycles without any damage, showing excellent mechanical robustness.

\section{Experimental section}

\subsection{Chemicals and materials}

Analytical grade silver nitrate $\left(\mathrm{AgNO}_{3}\right)$, polyvinylpyrrolidone (PVP, K30), ferric chloride $\left(\mathrm{FeCl}_{3} \cdot 6 \mathrm{H}_{2} \mathrm{O}\right)$ and ethylene glycol (EG) were used as raw materials. The graphene ink (purchased from Suzhou Tanfeng Graphene Technology Co., Ltd.) contains $5 \mathrm{wt} \%$ graphene with a flake size of $1-2 \mu \mathrm{m}$ and thickness of 1$5 \mathrm{~nm}$.

\subsection{Synthesis of AgNWs}

AgNWs were synthesized by a facile polyol method as in a previous report. ${ }^{39}$ Firstly, $0.25 \mathrm{~g}$ of $\mathrm{AgNO}_{3}$ and $0.25 \mathrm{~g}$ of PVP were mixed in $25 \mathrm{~mL}$ of EG under magnetic stirring until completely dissolved. Then, $0.34 \mathrm{~g}$ of $\mathrm{FeCl}_{3}$ solution (concentration of $0.6 \mathrm{mM}$ ) in EG was injected into the above mixture via a syringe pump and further stirred for 5 minutes. Finally, the solution was put into a heated oven to synthesize AgNWs of various sizes. Three groups of parameters were used to obtain AgNWs: (1) $110{ }^{\circ} \mathrm{C}$ for 12 hours; (2) $130{ }^{\circ} \mathrm{C}$ for 4 hours; (3) $180{ }^{\circ} \mathrm{C}$ for 45 minutes, which were annotated as AgNW1, AgNW2 and AgNW3, respectively. The products were washed with water and ethanol three times, and collected by natural precipitation. The AgNWs were dispersed in ethanol to form Ag ink with a mass fraction of $1 \%$.

\subsection{Fabrication of AgNW-G hybrid conductive tracks}

The standard fabrication process is shown in Fig. 1. The Ag ink was injected into commercial graphene ink at different mass ratios and under ultrasonic vibration for 10 minutes to achieve homogeneous AgNW-G hybrid inks. Then the AgNW-G inks were deposited on PET substrates by a screen coating method. The size of the conductive tracks was $4 \times 20 \mathrm{~mm}$, which was controlled by the yellow tapes. The ink-coated PET films were further heated in an oven over a temperature range from $100{ }^{\circ} \mathrm{C}$ to $250{ }^{\circ} \mathrm{C}$ for 15 minutes to improve the conductivity. Highly conductive Ag-G tracks were obtained after the thermal sintering process.

\subsection{Characterization}

The phase of the as-synthesized AgNWs and purchased graphene ink was confirmed by X-ray diffractometry (XRD, Miniflex600, Rigaku Corporation). The morphologies of AgNWs and Ag-G hybrid conductive tracks were characterized by scanning electron microscopy (SEM, SU1510, Hitachi High Technologies America Inc.). The microstructure of the AgNWs was checked with a transmission electron microscope (TEM, Tecnai G2 F30, FEI Technologies Inc.) operated at $300 \mathrm{kV}$. The thermal behavior of the commercial graphene ink was investigated by thermogravimetric analysis/differential scanning calorimetry (TGA/DSC, LF 1600, METTLER TOLEDO) at a heating rate of $10{ }^{\circ} \mathrm{C} \mathrm{min}$ under air atmosphere after being pre-dried at $60{ }^{\circ} \mathrm{C}$. The sheet resistance was examined by a four-probe method with a surface resistivity meter (RTS-9, Guangzhou Four-Point Probes Technology Co. Ltd). For each resistivity measurement, we prepared at least 3 samples and 3 points were tested for each sample. To test the electrical property during the bending process, we bent the AgNW-G hybrid tracks around cylinders with different diameters from 10 to $20 \mathrm{~mm}$. The ends of the samples were coated with $\mathrm{Ag}$ inks and stuck with $\mathrm{Cu}$ tape to facilitate the measurement of resistance by a multimeter (U1242A, Agilent Technologies). The AgNW-G hybrid conductive tracks were used to illuminate light emitting diodes (LEDs) which were connected to a power source (KRP-305D, Shenzhen Zhaoxin Electronic Instruments Equipment Co. Ltd) to input a DC voltage of $3 \mathrm{~V}$.

\section{Results and discussion}

The XRD spectrum of long AgNWs is shown in Fig. 2(a). The diffraction peaks at $38.1,44.1,64.4$ and $77.3^{\circ}$ correspond to the $\{111\},\{200\},\{220\}$ and $\{311\}$ planes of face-centered cubic (FCC) $\mathrm{Ag}$ (JCPDS 4-0783), agreeing well with the previous literature. ${ }^{\mathbf{4 0 , 4 1}}$ No other peaks can be observed, demonstrating the high purity of the products. The high resolution TEM (HRTEM) image (Fig. 2(b)) also confirms the high crystallinity of the AgNWs. A regular lattice fringe can be clearly observed. The lattice distance of $0.236 \mathrm{~nm}$ corresponds to the planar spacing of the

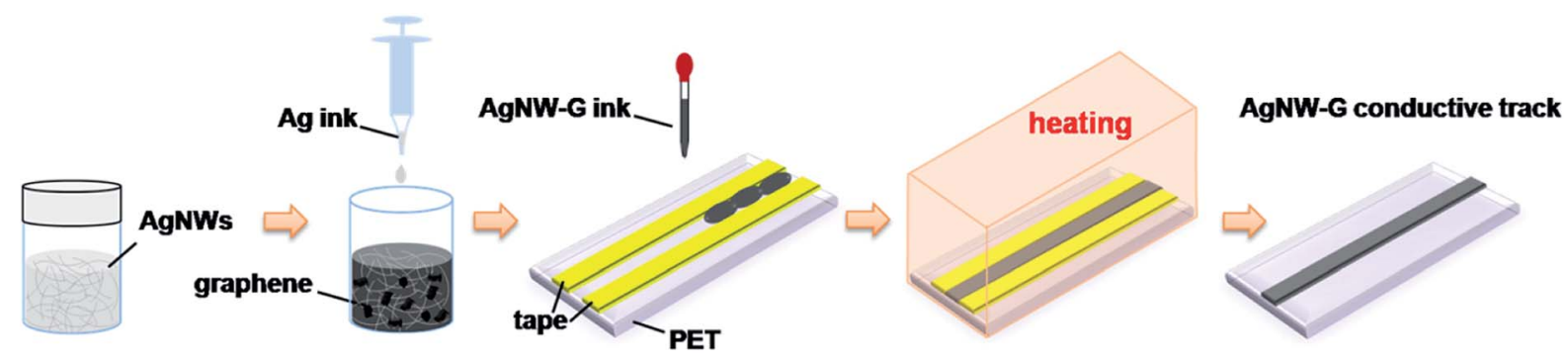

Fig. 1 Fabrication process of AgNW-G hybrid conductive tracks. 

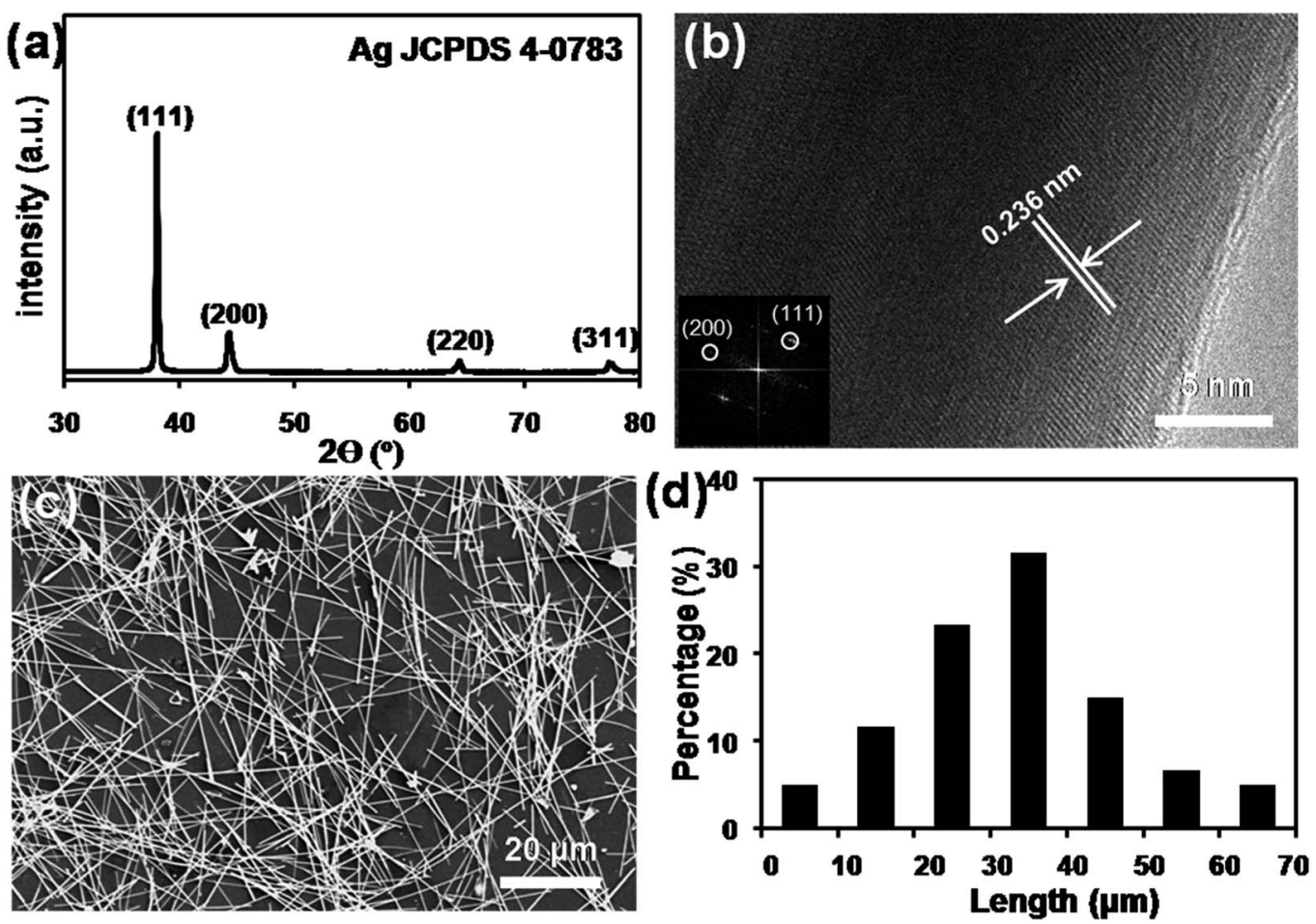

Fig. 2 (a) XRD pattern, (b) HRTEM image, (c) SEM image and (d) length distribution of as-synthesized AgNWs. Inset in (b) shows the fast Fourier transform (FFT) pattern of the HRTEM image.

(111) plane of FCC Ag. The morphological image of AgNWs checked by SEM (Fig. 2(c)) indicates good dispersion. It was found that there were still PVP residuals on the surface of AgNWs (Fig. S1 $\dagger$ ) which were not washed away totally due to the strong adhesion between PVP and AgNWs. ${ }^{42}$ The thin PVP layer adsorbed on the AgNWs acts as an electrically insulating barrier at the junctions with other AgNWs or graphene sheets. To obtain the length distribution of the AgNWs, we randomly chose 70 AgNWs from the SEM image and measured their lengths. The length of the AgNWs is mostly distributed between 20 and $50 \mu \mathrm{m}$. The average length of the AgNWs is calculated to be $32 \mu \mathrm{m}$, which is much longer than that of AgNWs (around 10 $\mu \mathrm{m})$ in previous reports. ${ }^{43}$

The as-synthesized AgNWs were mixed with commercial graphene ink and printed on PET substrates, and thermally sintered at $150{ }^{\circ} \mathrm{C}$ for 15 minutes. The XRD pattern of the AgNW-G tracks is presented in Fig. 3(a) along with those of AgNWs and graphene inks. The diffraction peaks of the AgNW$\mathrm{G}$ tracks were consistent with an $\mathrm{Ag}$ and graphene composite. Although sintered at $150{ }^{\circ} \mathrm{C}$ for 15 minutes, no peaks of $\mathrm{Ag}$ oxides in the final AgNW-G tracks can be found. The SEM images of sintered samples are shown in Fig. 3(b-d). As shown in Fig. 3(b), the AgNWs are uniformly dispersed in the graphene base without aggregation. The graphene flakes lay flat on the substrates and cover the AgNWs (Fig. S2 $\dagger$ ). The size of the graphene flakes is only 1-2 $\mu \mathrm{m}$, which is much smaller than the length of the AgNWs. A long AgNW acts as a cable and is able to connect tens of graphene flakes, which is assumed to improve the conductivity by building electrical channels among different graphene flakes. Meanwhile, the AgNW network with high conductivity also contributes to the outstanding performance of the AgNW-G hybrid tracks. On the other hand, the AgNWs fill the interlamellar spacing of the graphene flakes, which provides interpass electron-transport paths between the graphene layers. As shown in Fig. 3(c), there is a discrepancy in the contrast of the AgNWs, indicating that NW-1 and NW-3 are sandwiched between different graphene flakes. After sintering, the graphene flakes closely stack to form a layered structure, as shown in Fig. 3(d), while the randomly wrapped AgNWs are prone to disperse between the layers. ${ }^{36}$ The force exerted by the graphene appears to compress the AgNWs into a more planar structure. ${ }^{44}$ Most AgNWs form a planar network between the layers, which facilitates the electron transport between graphene layers. There are still some AgNWs passing across the graphene layers on account of the smaller size of the graphene sheets which cannot fully cover the long AgNWs. For example, considering the gradually varied grayscale, NW-2 is seen to be partially buried under the graphene flakes, in which the top part is exposed and the bottom part is covered by the graphene flakes. The typical morphology is also found in other AgNW-G hybrid tracks containing various amounts of Ag (Fig. S3†). The AgNWs randomly insert among or across graphene flakes. The gaps between the graphene layers are connected by the AgNWs which are either inserted into the graphene layer or pass through the graphene flakes (marked by the white arrows). The AgNWs fill the interlamellar spacing of the graphene flakes and bridge them, which provides interpass electron-transport paths between the graphene layers. ${ }^{45}$ From above, the AgNWs form a 3D conductive network in the graphene base, which is expected to offer a better conductivity for the AgNW-G hybrid 

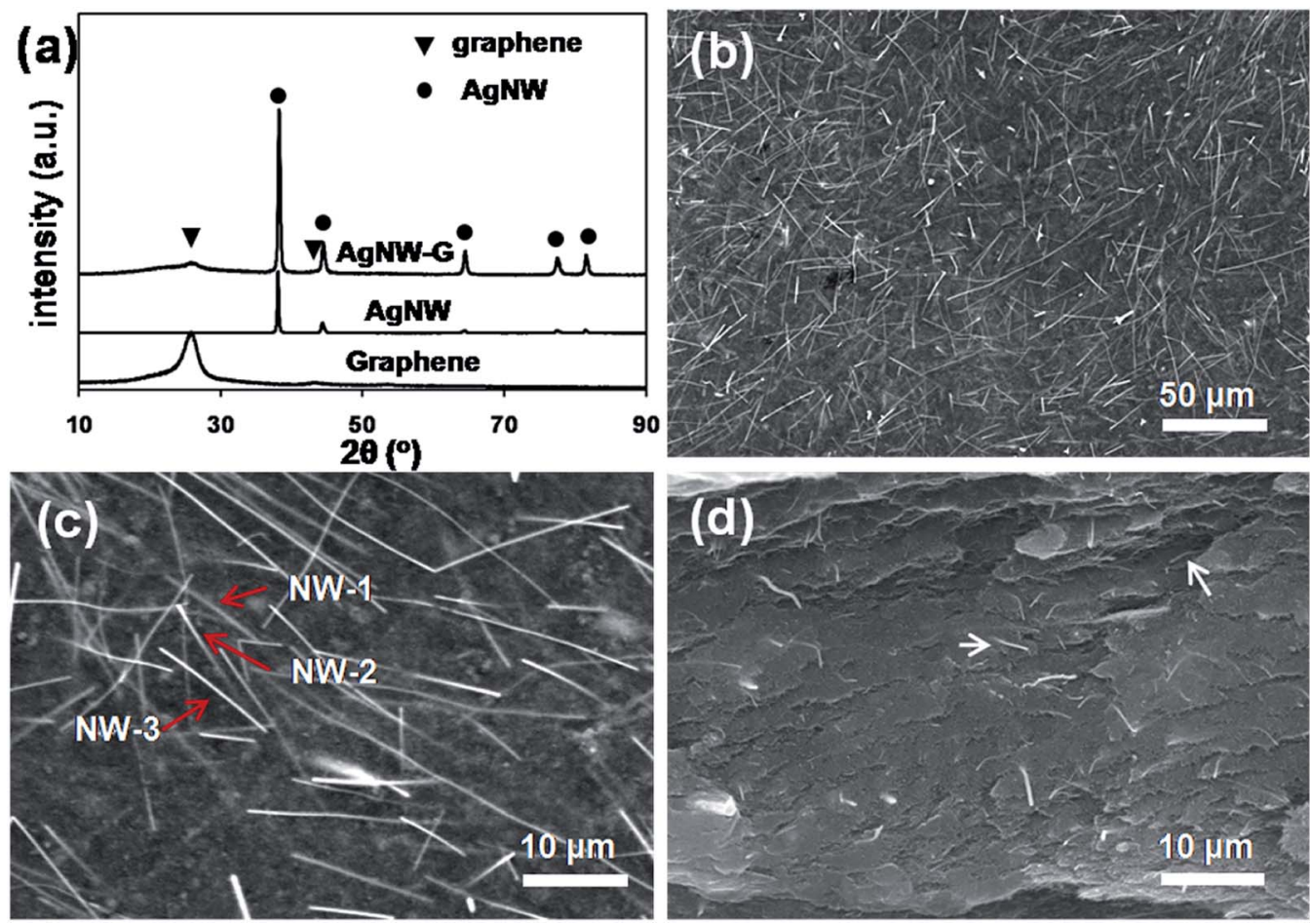

Fig. 3 (a) XRD pattern of AgNW-G tracks; (b and c) top view and (d) tilted view of AgNW-G hybrid tracks with 20 wt\% AgNWs after sintering at $150^{\circ} \mathrm{C}$ for 15 minutes.

tracks. In fact, pure graphene tracks with a resistance of 120 $\mathrm{m} \Omega \mathrm{cm}$ can be achieved by a simple sintering method at $150^{\circ} \mathrm{C}$. The resistance mainly depends on the gaps and boundaries between the graphene flakes, which could be connected by AgNWs in the composite inks. The resistivity of the AgNW-G hybrid track is reduced to $8.6 \mathrm{~m} \Omega \mathrm{cm}$ by adding $20 \mathrm{wt} \%$ AgNWs, which is 14 times better than that of pure graphene tracks. As a comparison, the electrical conductivity of AgNWs/graphene papers shows a 3-fold increase with respect to the pure graphene reported by Chen. ${ }^{36}$ The long AgNW shows superiority in hybridizing graphene for preparing highly conductive tracks.

In order to further study the function of AgNWs, AgNW-G hybrid tracks with different amounts of AgNWs were prepared and the corresponding resistivities were examined. Fig. 4 shows the microstructure and electrical property of AgNW-G hybrid tracks containing various amounts of $\mathrm{Ag}$. It can be seen from the SEM images that the density of AgNW is increased with a greater loading of AgNWs. When only $5 \mathrm{wt} \%$ AgNWs are added into the graphene ink, the junctions between AgNWs are fewer. Even so, the resistivity of the resultant AgNW-graphene hybrid tracks is sharply decreased to $26.7 \mathrm{~m} \Omega \mathrm{cm}$ which is about $1 / 5$ that of a pure graphene track. By further enlarging the AgNW content to $10 \mathrm{wt} \%$ and $15 \mathrm{wt} \%$, the resistivities of the AgNW-G hybrid tracks decrease to 17.0 and $14.3 \mathrm{~m} \Omega \mathrm{cm}$, respectively. More AgNWs create more conductive channels to connect graphene flakes and show more connections in AgNW networks, both of which contribute to the better performance of the AgNW-G hybrid tracks. When the amount of $\mathrm{AgNWs}$ is increased to $20 \mathrm{wt} \%$ and $30 \mathrm{wt} \%$, dense $\mathrm{AgNW}$ networks form in the graphene base. Thanks to the high conductivity of $\mathrm{Ag}$, the resistivities of the AgNW-G hybrid tracks decrease to 8.6 and $6.2 \mathrm{~m} \Omega \mathrm{cm}$. When the content of AgNWs is as low as $5 \mathrm{wt} \%$, the 3D network is not completed and only a small part of the graphene flakes are connected by the AgNWs. The AgNWs form effective pathways and lower the contact resistance between graphene flakes at contents of $10 \mathrm{wt} \%$ and $15 \mathrm{wt} \%$. The resulting resistivity decreases rapidly with an increase in the amount of AgNWs. When the content of AgNWs reaches $20 \mathrm{wt} \%$ and $30 \mathrm{wt} \%$, the 3D AgNW network is well connected and fully integrated with the graphene flakes. Meanwhile, AgNW-G hybrid tracks with excellent electrical performance are achieved. These results confirm that conductivity improvement is contributed by the Ag networks, and that a higher content of AgNWs leads to higher conductivity for AgNW-G hybrid tracks. Compared with other complex treatments, including high temperature annealing, vacuum filtration and drying, the fabrication process of the AgNWG tracks in our work only refers to facile drop coating and lowtemperature sintering for a short duration. The conductivity of AgNW-G hybrid tracks is much better than that of pure graphene films ${ }^{24}$ and comparable with other metal nanowire and graphene composites. . $^{35,36,38,45}$

The high conductivity of the AgNW-G hybrid tracks is presumably induced by the presence of long AgNWs. To demonstrate the dominant role of long AgNWs, hybrid tracks were prepared from graphene ink hybridized with AgNWs of various lengths. Three types of AgNWs were synthesized by adjusting the parameters during the polyol reaction. The SEM images of the AgNWs are shown in Fig. S4 $\dagger$ and the length distributions of these AgNWs were also collected. The average lengths of AgNW1, AgNW2 and AgNW3 were calculated to be 32, 18, and $12 \mu \mathrm{m}$, respectively. The SEM images of sintered AgNW-G 

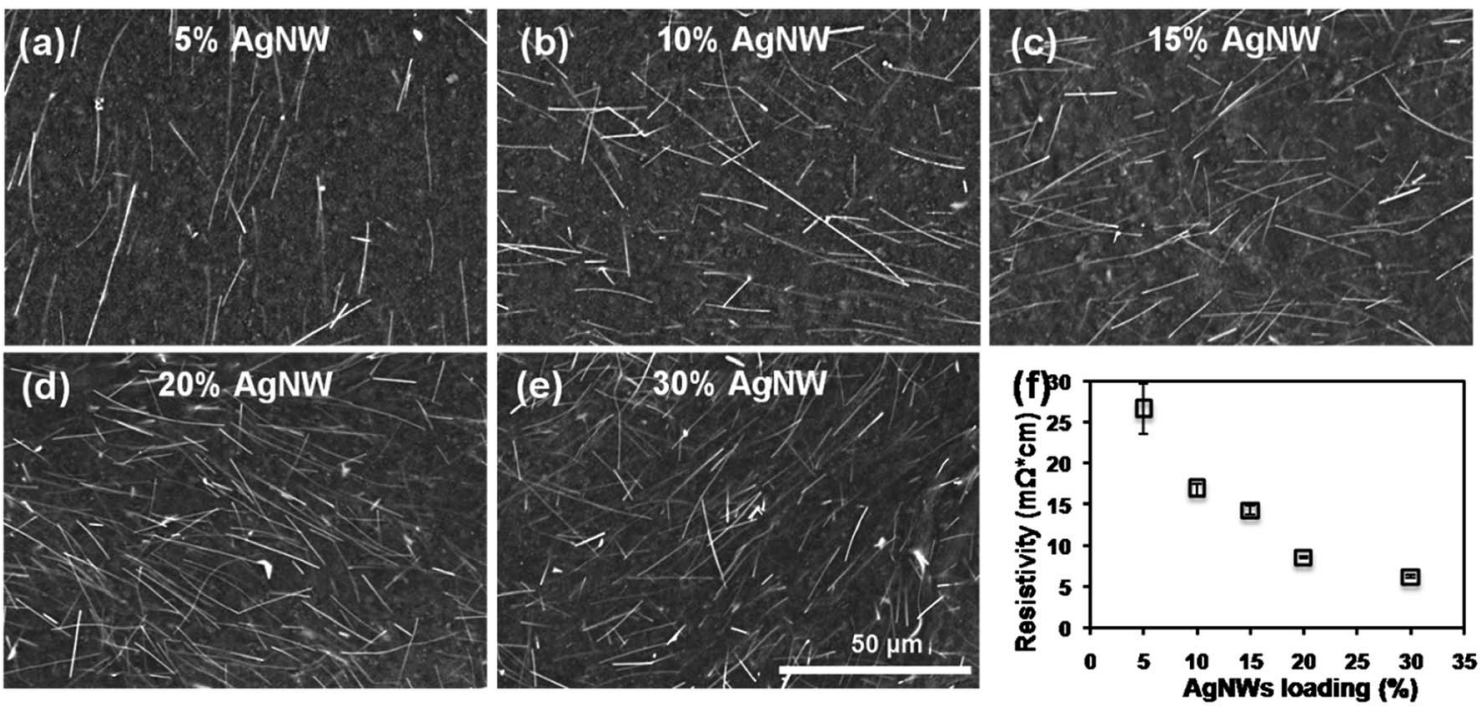

Fig. 4 SEM images of AgNW-G hybrid tracks with AgNWs loading of (a) 5 wt\%, (b) 10 wt\%, (c) 15 wt\%, (d) 20 wt\%, and (e) 30 wt\%. (f) Resistivity evolution of sintered AgNW-G tracks as a function of AgNWs loading.

hybrid tracks using AgNW1, AgNW2 and AgNW3 are shown in Fig. 5. It can be seen the AgNWs are uniformly dispersed in the graphene base except for AgNW3 which shows a bundled structure due to the rapid reaction at high temperature during the synthetic process. The lowest resistivity of $12.3 \mathrm{~m} \Omega \mathrm{cm}$ is obtained for the AgNW1-G tracks sintered at $100{ }^{\circ} \mathrm{C}$. The long AgNW1 can connect a large quantity of graphene flakes without interruption and build more effective channels among the graphene flakes than those of short AgNW2 and AgNW3 ${ }^{44}$ Moreover, the relatively few contact points between long AgNW1 reduced the unavoidable contact resistance in the AgNW network. ${ }^{39,46,47}$ While the resistivities of
AgNW2-G and AgNW3-G tracks are 22.4 and $33.6 \mathrm{~m} \Omega \mathrm{cm}$ with the same addition of AgNWs, respectively. It should be mentioned that despite the similar lengths of AgNW2 and AgNW3, the conductivity of the AgNW2-G track is much better than that of the AgNW3-G track, which indicates that the bundled AgNW3 cannot effectively connect the graphene flakes. This also suggests that the AgNWs work as cables and bridges to connect the graphene flakes in the hybrid tracks.

The influence of sintering temperature on the conductivity of the AgNW-G hybrid tracks was also studied. The resistivity evolution of sintered AgNW-graphene patterns as a function of
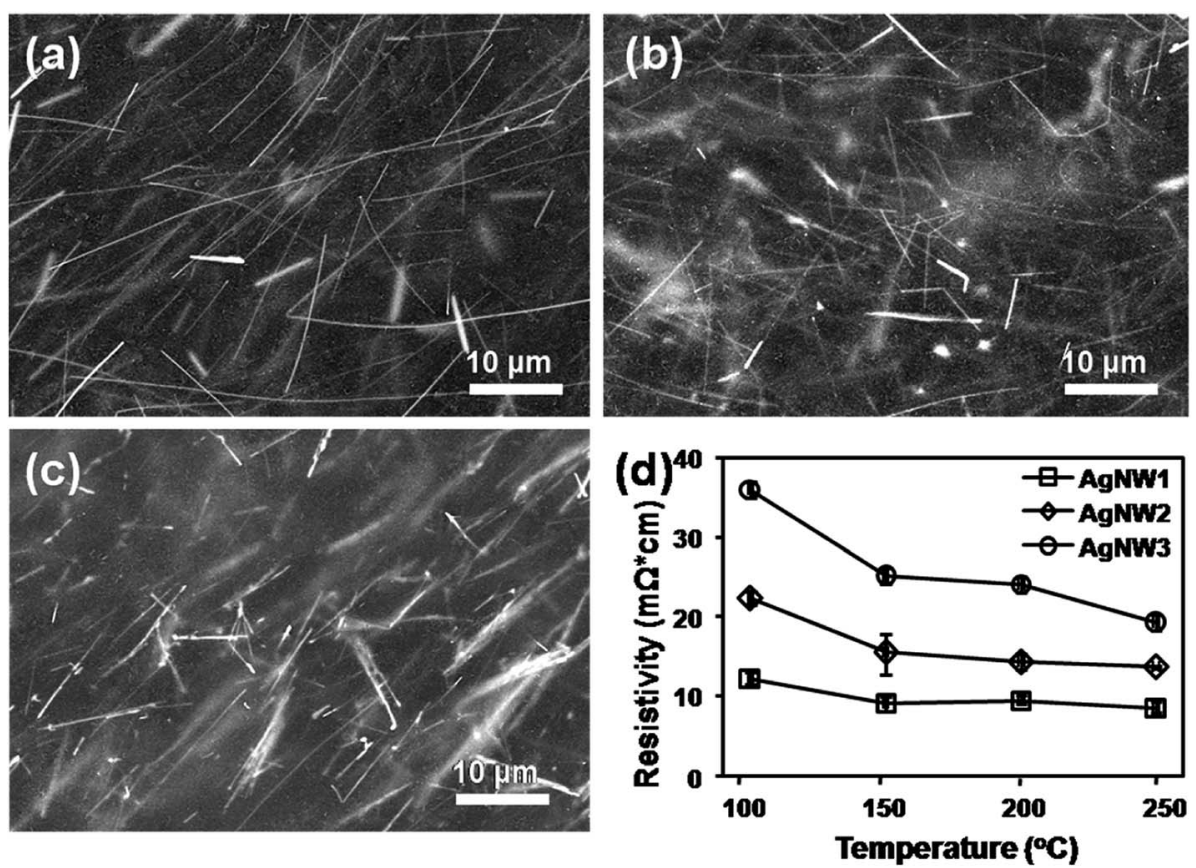

Fig. 5 SEM images of AgNW-G hybrid conductive tracks with 20 wt\% AgNW using (a) AgNW1, (b) AgNW2, and (c) AgNW3, respectively. (d) Resistivity evolution of sintered AgNW-G patterns as a function of sintering temperature. 
sintering temperature is plotted in Fig. 5(d). It can be seen that the resistivities of all three AgNW-G tracks decrease with increasing temperature. The TGA result for graphene ink (Fig. S5 $\dagger$ ) shows no obvious weight change below $250{ }^{\circ} \mathrm{C}$, indicating that the solvents in the commercial ink are completely removed at low temperatures, even at $60{ }^{\circ} \mathrm{C}$ over a long period. Hence, $100{ }^{\circ} \mathrm{C}$ is high enough for the removal of solvents and the formation of the graphene layer structure. The improvement in conductivity for the AgNW-G hybrid tracks at high temperatures can be ascribed to the enhanced joining between the AgNWs ${ }^{48,49}$ and the promoted connections between AgNW and graphene. It was reported that thermal annealing can drastically lower the electrical resistance of the AgNW networks from $10^{-7} \Omega \mathrm{sq}^{-1}$ to $9.5 \Omega \mathrm{sq}^{-1} \cdot{ }^{48}$ The thermal sintering promotes the diffusion of $\mathrm{Ag}$ atoms and decomposes the organic residues on the surface of AgNWs, both of which benefit the joining between AgNWs. ${ }^{48,50}$ On the other hand, the graphene wraps the surface cleaned AgNWs tightly at higher temperatures, resulting in more intimate AgNW to graphene contact. The improved connections of AgNWs to homogeneous AgNWs and heterogeneous graphene greatly decrease the contact resistance at the cross spots, which dominates the resistance of the AgNW-G composite. The electrical resistance of the AgNW-G tracks decreases sharply at $150{ }^{\circ} \mathrm{C}$ and continues to decrease slowly below $250{ }^{\circ} \mathrm{C}$. During the thermal sintering process at various temperatures, the AgNWs maintain the network structure in the graphene base without destruction, as shown in Fig. S6. $\dagger$ The resistivity evolution also demonstrates that the synergy between AgNWs and graphene effectively improves the electrical property of the AgNW-G hybrid tracks.

Fig. 6 illustrates the functions of AgNWs in the highly conductive AgNW-G hybrid tracks. The conductive pathways of pure graphene tracks with lots of boundaries and layer gaps are cut off. The boundaries severely limit the electronic transport. ${ }^{44}$ The AgNWs connect the graphene flakes, providing electrical channels for electrons among boundaries. Meanwhile, the AgNW bridges the gaps between layers by thoroughly filling in between two adjacent layers or passing through the graphene flakes. The filler AgNWs build possible channels for electron transport across graphene layers. Furthermore, the AgNWs form a highly conductive 3D network. Based on these mechanisms, highly conductive AgNW-G hybrid tracks are successfully obtained by a facile solution-based drop coating and low temperature sintering process.
The electromechanical property is essential for realizing the application of AgNW-G hybrid tracks to flexible electronics. The AgNW-G hybrid tracks were bent around bottles with different diameters and the resistances were recorded after each bending. Fig. 7(a) presents the relative resistance change of the AgNW-G tracks with various bending diameters. The resistances of the AgNW-G track are increased to 1.62, 1.34, 1.17, 1.06 and 1.03 times when bent with diameters of 5, 10, 15, 20 and $25 \mathrm{~mm}$, respectively. This could be interpreted as the bending stress causing strains in the AgNW-G tracks, which would break the conductive paths in the tracks. ${ }^{47,51}$ Delightfully, after release, the relative resistance reduces to almost 1 for every diameter except for $5 \mathrm{~mm}$, indicating that the conductive pathways recover. The relative resistance of the AgNW-G tracks increases to 1.1 even under releasing status after 20 cycles of the bending and releasing process. When the AgNW-G tracks were bent around a pencil with a diameter of $5 \mathrm{~mm}$, the resistance continued to grow slowly with more bending cycles, since the macroscopic cracks were generated without recovery. To check the long-term reliability, an AgNW-G track (length $=50 \mathrm{~mm}$ and width $=4$ $\mathrm{mm}$ ) was bent and released for 100 cycles with a bending radius of $10 \mathrm{~mm}$, as shown in Fig. 7(b). No significant change in the relative resistance can be found even after 100 bending cycles. The experiments imply that the AgNW-G hybrid tracks possess superior reliability under mechanical stress, which qualifies them for flexible electronics.

The application of AgNW-G hybrid tracks is demonstrated to light up an LED during bending, twisting and adhesive tape tests, as shown in Fig. 8(a-d). The LED illumination intensity remains constant without visible degradation, which signifies that the AgNW-G inks are desirable for flexible printed electronic devices. To take advantage of printed electronics, the AgNW-G hybrid ink was also coated on photographic paper with a pattern of the Hangzhou Dianzi University logo and used as part of an LED circuit (Fig. 8(e and f)). Similar to PET films, the AgNW-G coated photographic paper can also light up the LED when the paper is bent, showing that the AgNW-G ink can be used as a universal coating on various flexible substrates. In respect of the facile fabrication process, high conductivity and mechanical robustness, the high-performance AgNW-G hybrid track is expected to be applicable to various electronics other than an LED circuit, and may have potential applications in the development of printed electronics.

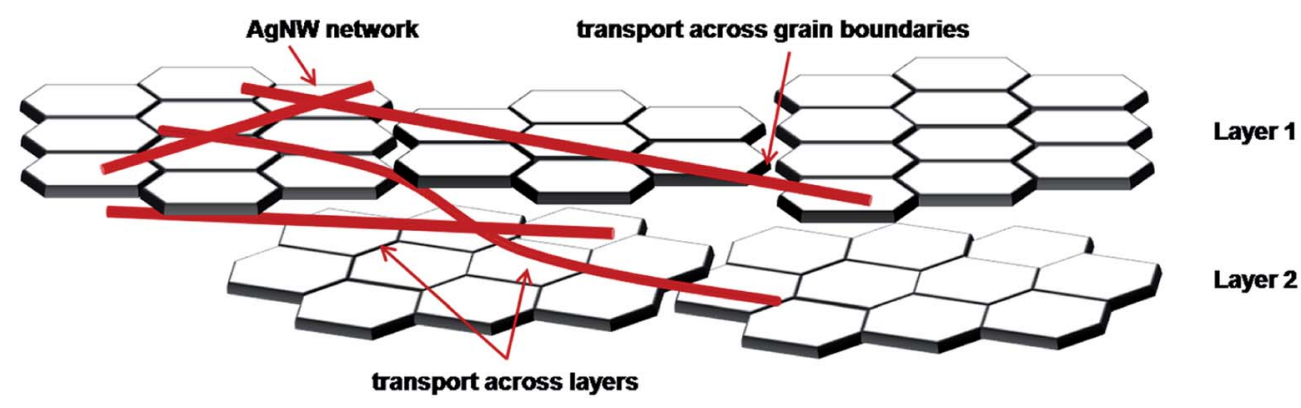

Fig. 6 Schematic illustration of the conductive long AgNW channels in the graphene base. 

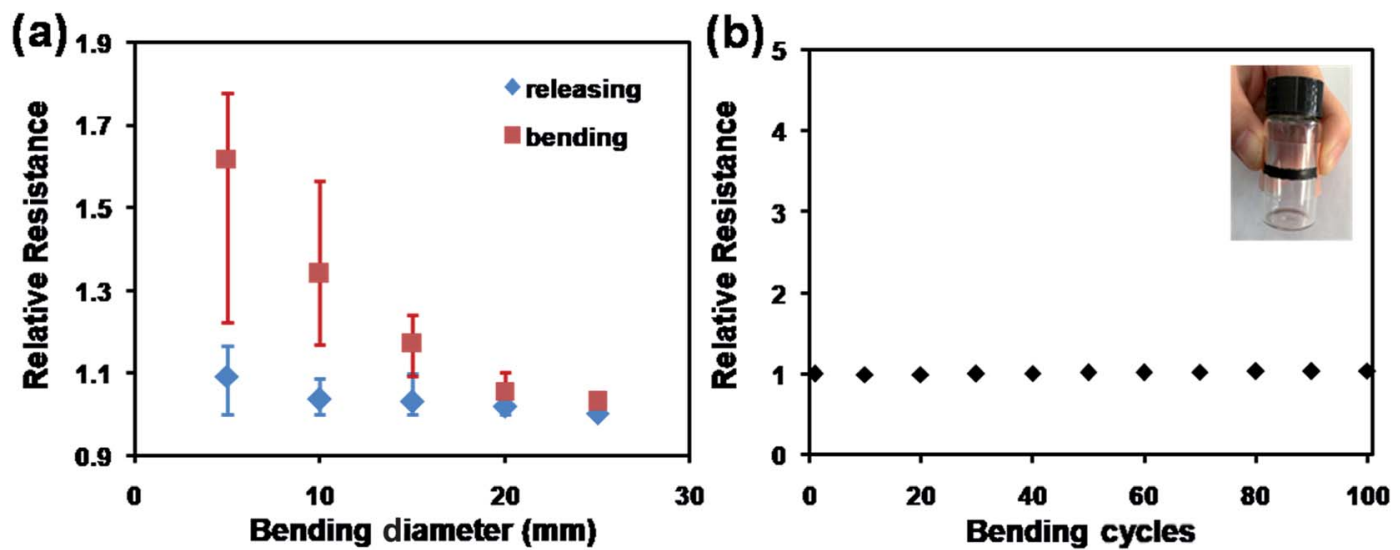

Fig. 7 Relative resistance changes of AgNW-G tracks (a) after 20 cycles of a bending process around cylinders with different diameters, (b) during 100 cycles of bending and releasing. Inset in (b) shows a photograph of the AgNW-G hybrid tracks around a glass bottle.
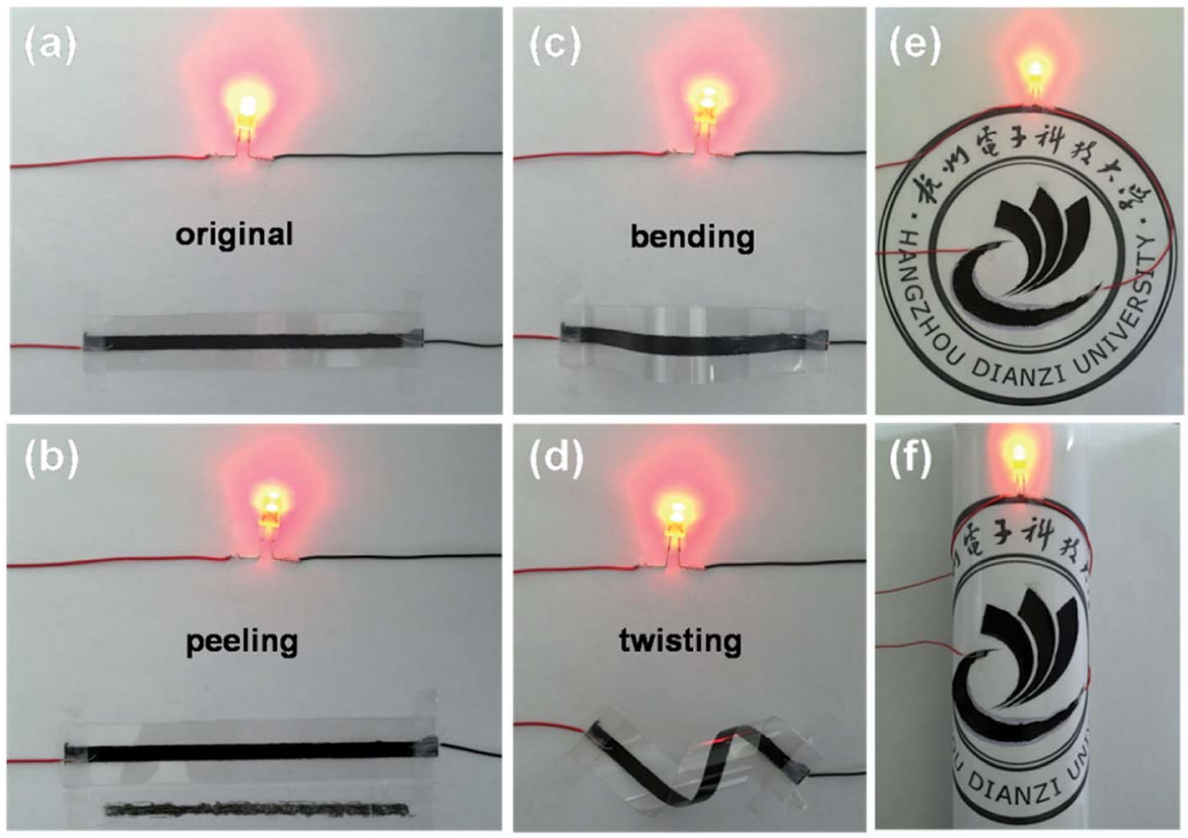

Fig. 8 Photographs of the LED circuit with a $45 \mathrm{~mm}$-long AgNW-G hybrid trackson PET at (a) original status and during (b) peeling, (c) bending, and (d) twisting tests. Photos of AgNW-G hybrid conductive patterns with the shape of the Hangzhou Dianzi University logo on a paper substrate as part of an LED circuit under (e) natural and (f) bending status.

\section{Conclusions}

Long AgNWs and graphene inks were hybridized to prepare highly conductive AgNW-G tracks by a thermal sintering method. The AgNWs greatly improve the conductivity since the long AgNWs effectively connect graphene flakes without interruption and form a more conductive 3D network compared to short AgNWs. Highly conductive hybrid AgNW-G tracks with a resistivity of $8.6 \mathrm{~m} \Omega \mathrm{cm}$ have been achieved by adding $20 \mathrm{wt} \%$ AgNWs at $150{ }^{\circ} \mathrm{C}$ for only $15 \mathrm{~min}$. The AgNW-G tracks show superior flexibility even after 100 cycles of a bending process. The AgNW-G hybrid ink with excellent mechanical robustness shows great potential in the application of flexible circuits.

\section{Conflicts of interest}

There are no conflicts to declare.

\section{Acknowledgements}

The authors are grateful for financial support from the National Natural Science Foundation of China (Grant No. 51705115).

\section{References}

1 S. H. Kim, K. Hong, W. Xie, K. H. Lee, S. Zhang, T. P. Lodge and C. D. Frisbie, Adv. Mater., 2013, 25, 1822-1846.

2 J. A. Lewis and B. Y. Ahn, Nature, 2015, 518, 42-43. 
3 W. J. Hyun, E. B. Secor, M. C. Hersam, C. D. Frisbie and L. F. Francis, Adv. Mater., 2015, 27, 109-115.

4 Y. Aleeva and B. Pignataro, J. Mater. Chem. C, 2014, 2, 64366453.

5 A. Kamyshny and S. Magdassi, Small, 2014, 10, 3515-3535.

6 E. Roh, B. U. Hwang, D. Kim, B. Y. Kim and N. E. Lee, ACS Nano, 2015, 9, 6252-6261.

7 J. Lee, P. Lee, H. Lee, D. Lee, S. S. Lee and S. H. Ko, Nanoscale, 2012, 4, 6408-6414.

8 J. Viventi, D. H. Kim, L. Vigeland, E. S. Frechette, J. A. Blanco, Y. S. Kim, A. E. Avrin, V. R. Tiruvadi, S. W. Hwang, A. C. Vanleer, D. F. Wulsin, K. Davis, C. E. Gelber, L. Palmer, J. Van der Spiegel, J. Wu, J. Xiao, Y. Huang, D. Contreras, J. A. Rogers and B. Litt, Nat. Neurosci., 2011, 14, 1599-1605.

9 J. Liang, L. Li, X. Niu, Z. Yu and Q. Pei, Nat. Photonics, 2013, 7, 817-824.

$10 \mathrm{X}$. Yu, W. Zhang, P. Zhang and Z. Su, Biosens. Bioelectron., 2017, 89, 72-84.

11 Y. Wei, S. Chen, F. Li, Y. Lin, Y. Zhang and L. Liu, ACS Appl. Mater. Interfaces, 2015, 7, 14182-14191.

12 N. Kim, S. Kee, S. H. Lee, B. H. Lee, Y. H. Kahng, Y. R. Jo, B. J. Kim and K. Lee, Adv. Mater., 2014, 26, 2268-2272.

13 T. Kim, S. Ha, H. Choi, K. Uh, U. Kundapur, S. Park, C. W. Lee, S.-h. Lee, J. Kim and J.-M. Kim, Adv. Mater., 2017, 9, 19231-19237.

14 J. S. Kang, H. S. Kim, J. Ryu, H. Thomas Hahn, S. Jang and J. W. Joung, J. Mater. Sci.: Mater. Electron., 2010, 21, 1213-1220.

15 B. J. Perelaer, A. W. M. de Laat, C. E. Hendriks and U. S. Schubert, J. Mater. Chem., 2008, 18, 3209.

16 W. Shen, X. Zhang, Q. Huang, Q. Xu and W. Song, Nanoscale, 2014, 6, 1622-1628.

17 J. Perelaer, P. J. Smith, D. Mager, D. Soltman, S. K. Volkman, V. Subramanian, J. G. Korvink and U. S. Schubert, J. Mater. Chem., 2010, 20, 8446.

18 W. Li, H. Zhang, Y. Gao, J. Jiu, C. F. Li, C. Chen, D. Hu, Y. Goya, Y. Wang, H. Koga, S. Nagao and K. Suganuma, J. Mater. Chem. C, 2017, 5, 1155-1164.

19 K. Kordas, T. Mustonen, G. Toth, H. Jantunen, M. Lajunen, C. Soldano, S. Talapatra, S. Kar, R. Vajtai and P. M. Ajayan, Small, 2006, 2, 1021-1025.

20 O. S. Kwon, H. Kim, H. Ko, J. Lee, B. Lee, C. H. Jung, J. H. Choi and K. Shin, Carbon, 2013, 58, 116-127.

21 L. Huang, Y. Huang, J. Liang, X. Wan and Y. Chen, Nano Res., 2011, 4, 675-684.

22 N. Karim, S. Afroj, A. Malandraki, S. Butterworth, C. Beach, M. Rigout, K. S. Novoselov, A. J. Casson and S. G. Yeates, J. Mater. Chem. C, 2017, 5, 11640-11648.

23 A. A. Balandin, S. Ghosh, W. Z. Bao, I. Calizo, D. Teweldebrhan, F. Miao and C. N. Lau, Nano Lett., 2008, 8, 902-907.

24 C. L. Lee, C. H. Chen and C. W. Chen, Chem. Eng. J., 2013, 230, 296-302.

25 J. J. Jia, X. Y. Sun, X. Y. Lin, X. Shen, Y. W. Mai and J. K. Kim, ACS Nano, 2014, 8, 5774-5783.
26 X. Yu, D. Lin, P. Li and Z. Su, Sol. Energy Mater. Sol. Cells, 2017, 172, 252-269.

27 X. Zhao, P. Zhang, Y. Chen, Z. Su and G. Wei, Nanoscale, 2015, 7, 5080-5093.

28 E. B. Secor, B. Y. Ahn, T. Z. Gao, J. A. Lewis and M. C. Hersam, Adv. Mater., 2015, 27, 6683-6688.

29 P. G. Karagiannidis, S. A. Hodge, L. Lombardi, F. Tomarchio, N. Decorde, S. Milana, I. Goykhman, Y. Su, S. V. Mesite, D. N. Johnstone, R. K. Leary, P. A. Midgley, N. M. Pugno, F. Torrisi and A. C. Ferrari, ACS Nano, 2017, 11, 2742-2755.

30 H. W. Tien, Y. L. Huang, S. Y. Yang, J. Y. Wang and C. C. M. Ma, Carbon, 2011, 49, 1550-1560.

31 Z. Xu, H. Gao and G. Hu, Carbon, 2011, 49, 4731-4738.

32 J. H. Park, M. J. Park and J. S. Lee, Nanoscale, 2017, 9, 555-561.

33 P. Liu, Q. Tang, H. Liu and A. Lu, RSC Adv., 2017, 7, 1522815235.

34 Y. Li, P. Zhang, Z. Ouyang, M. Zhang, Z. Lin, J. Li, Z. Su and G. Wei, Adv. Funct. Mater., 2016, 26, 2122-2134.

35 D. Deng, S. Feng, M. Shi and C. Huang, J. Mater. Sci.: Mater. Electron., 2017, 28, 15411-15417.

36 J. Chen, H. Bi, S. Sun, Y. Tang, W. Zhao, T. Lin, D. Wan, F. Huang, X. Zhou, X. Xie and M. Jiang, ACS Appl. Mater. Interfaces, 2013, 5, 1408-1413.

37 L. Y. Xu, G. Y. Yang, H. Y. Jing, J. Wei and Y. D. Han, Nanotechnology, 2014, 25, 055201.

38 S. Chen, Y. Wei, S. Wei, Y. Lin and L. Liu, ACS Appl. Mater. Interfaces, 2016, 8, 25563-25570.

39 J. Jiu, T. Araki, J. Wang, M. Nogi, T. Sugahara, S. Nagao, H. Koga, K. Suganuma, E. Nakazawa, M. Hara, H. Uchida and K. Shinozaki, J. Mater. Chem. A, 2014, 2, 6326-6330.

40 Y. Sun and Y. Xia, Adv. Mater., 2002, 14, 833-937.

41 Y. Sun, Y. Yin, B. T. Mayers, T. Herricks and Y. Xia, Chem. Mater., 2002, 14, 4736-4745.

42 J. Wang, J. Jiu, T. Araki, M. Nogi, T. Sugahara, S. Nagao, H. Koga, P. He and K. Suganuma, Nano-Micro Lett., 2014, 7, 51-58.

43 S. M. Bergin, Y. H. Chen, A. R. Rathmell, P. Charbonneau, Z. Y. Li and B. J. Wiley, Nanoscale, 2012, 4, 1996-2004.

44 R. Chen, S. R. Das, C. Jeong, M. R. Khan, D. B. Janes and M. A. Alam, Adv. Funct. Mater., 2013, 23, 5150-5158.

45 Y. Zhu, Y. Hu, P. Zhu, T. Zhao, X. Liang, R. Sun and C.-p. Wong, New J. Chem., 2017, 41, 4950-4958.

46 S. Wang, Y. Tian, S. Ding and C. Wang, Mater. Res. Express, 2016, 3, 075007.

47 P. Lee, J. Lee, H. Lee, J. Yeo, S. Hong, K. H. Nam, D. Lee, S. S. Lee and S. H. Ko, Adv. Mater., 2012, 24, 3326-3332.

48 D. P. Langley, M. Lagrange, G. Giusti, C. Jiménez, Y. Bréchet, N. D. Nguyen and D. Bellet, Nanoscale, 2014, 6, 13535-13543.

49 I. E. Stewart, M. J. Kim and B. J. Wiley, ACS Appl. Mater. Interfaces, 2017, 9, 1870-1876.

50 M. Lagrange, D. P. Langley, G. Giusti, C. Jimenez, Y. Bréchet and D. Bellet, Nanoscale, 2015, 7, 17410-17423.

51 W. Hu, R. Wang, Y. Lu and Q. Pei, J. Mater. Chem. C, 2014, 2, 1298-1305. 